\title{
FINGERPRINT BASED STUDENT ATTENDANCE SYSTEM WITH SMS ALERT TO PARENTS
}

\author{
K.Jaikumar ${ }^{1}$, M.Santhosh Kumar ${ }^{2}$, S.Rajkumar ${ }^{3}$, A.Sakthivel ${ }^{4}$ \\ ${ }^{1}$ Asst. Professor-ECE, P. A. College of Engineering and Technology \\ ${ }^{2}$ Student-ECE, P. A. College of Engineering and Technology \\ ${ }^{3}$ Student-ECE, P. A. College of Engineering and Technology \\ ${ }^{4}$ Student-ECE, P. A. College of Engineering and Technology
}

\begin{abstract}
This paper is a study of a fingerprint recognition system based on minutiae based fingerprint algorithms used in various techniques. This line of track mainly involves extraction of minutiae points from the model fingerprint images and fingerprint matching based on the number of minutiae pairings among two fingerprints. This paper also provides the design method of fingerprint based student attendance with help of GSM. This system ignores the requirement for stationary materials and personnel for keeping of records.
\end{abstract}

Keywords - GSM, LCD

\section{INTRODUCTION}

Fingerprints are one of the main forms in biometric field which used to identify the individual and their uniqueness. Fingerprint authentication is one of popular authentication systems in the world. Due to their consistency and uniqueness it is used widely. There are several sources available for the collection and their established use [1]. In the past, magnetic card attendance system was widely used. But, this system has lot of bugs in it. The card may be lost or damaged. This fingerprint attendance system is highly secure and cannot be forged easily. In recent time, there has been high level of impersonation experienced on a daily basis in both private and public sectors, the ghost worker syndrome which has become a menace across all tiers of government, employers concerns over the levels of employee absence in their workforce and the difficulty in managing student attendance during lecture periods. Fingerprints are a form of biometric identification which is unique and does not change in one's entire lifetime. This paper presents the attendance management system using fingerprint technology in a university environment. It consists of two processes namely; enrolment and authentication.

During enrolment, the fingerprint of the user is captured and its unique features extracted and stored in a database along with the users identity as a template for the subject. The unique features called minutiae points were extracted using the Crossing Number $(\mathrm{CN})$ method which extracts the ridge endings and bifurcations from the skeleton image by examining the local neighborhoods of each ridge pixel using a $3 \times 3$ window. During authentication, the fingerprint of the user is captured again and the extracted features compared with the template in the database to determine a match before attendance is made.
Attendance management system is one of the most advanced applications in biometric technology. It cannot be forged easily. With the integration and use of biometric technology getting simpler, many institutions are using down the biometric road to verify the time and attendance of their students and staffs. The system also contains a GMS Modem, which can be used to send the attendance information of the students automatically to their parents. The Embedded system using a small LCD user interface can be interfaced with the computer by using serial communication interface. The previous papers done were only the fingerprint based attendance system and a report generation. It does not have any SMS alert to the parents. This paper is to send SMS alert to parents by means of GSM.

The Fingerprint authentication has many advantages such as very high accuracy, the most economical biometric PC user authentication technique [2]. It is one of the safest biometric authentication methods widely used. It is very easy to use. Small storage space required for the biometric template, reducing the size of the database memory required and it is standardized. 


\section{GENERAL BLOCK DIAGRAM}

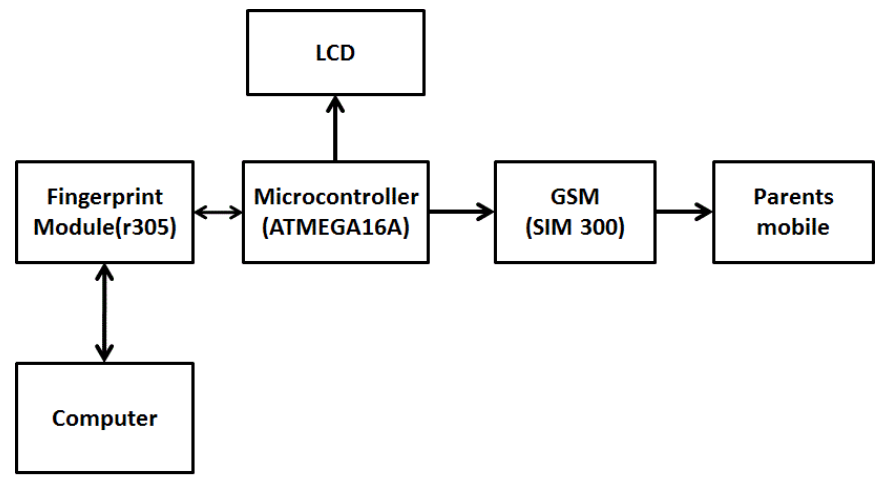

Fig 1 General block diagram

\section{BLOCK DIAGRAM EXPLANATION}

The finger prints from the various users are acquittedusing the fingerprint module. For example we are taking the samples of three or four fingerprints and they are enhanced using several enhancement techniques [3]. After that we detect the edges along the image using the edge detection function. Here we use the prewitt operator for the detecting the edges. We use minutiae matching algorithm for matching the finger print images. Instead of doing all these image processing works, we had used Fingerprint Module (R305) in this paper. The circuit is made to be switched ON and all the initialization processes are done.The "Initialization done" message has to be displayed in the screen.Up to that user should not keep any fingerprints for scanning.After that "Show the Finger" will be displayed on the screen. The fingerprint that has to be compared is already stored in the memory of fingerprint module. The fingerprint module is capable of storing about 100 images in the inbuilt memory.Now the fingerprints are kept for scanning with in a stipulated time period.Fingerprint module started to compare the results and it gives the hex codes to the microcontroller for further operations. The microcontroller starts to send the control to GSM based on the results from the finger print module.

But the microcontroller has only one transmitter pin in it.We have to communicate both of the GSM and fingerprint module but not at a time.For that we are using Relay for switching between the GSM and fingerprint module.There are also commands for holding the fingerprints and for comparing it.The person whose fingerprint is matched and there will not be any SMS send to those persons. The persons whose fingerprints were not kept were takenand SMS will be sent only to those numbers.If the fingerprint of an unknown person is kept for scanning then the scanning will not take place.If unknown persons fingerprint is kept then the message "Not Identified" will be displayed.With the help of this the parents can be known about their arrival of the Students to the college or school.The fingerprints are one of the greatest authentication system where students cannot forge very easily.This can be practically implemented by extending the time period and student's attendance can be managed.

\section{INTERNAL WORKING OF FINGERPRINT MODULE}

\subsection{Image Acquisition}

The Fingerprint images from various users are taken using the module. The finger module itself has an internal memory which can store about 100 images in it. These images are used for the enhancement in next stages.

\subsection{Image Enhancement}

The image is enhanced using the techniques like Histogram Equalization [4]. It is nothing but the graph plot for number of pixels against the gray level. The overall contrast of the image is made uniform and image looks enhanced. Now the image is suitable for the extraction of minutiae extraction.

\subsection{Edge Detection}

They are many operators used for detecting the edges. The operators are Prewitt, Laplacian, Sobels, Robertson Operators [5]. The Prewitt operator is one of the best edge detecting operator and we are implementingPrewitt operator in this paper. The edges have to be detected in order to match the input image with already saved image. There are two types of masking used here. They are

i) Horizontal masking

ii) Vertical masking

Edges are calculated by using difference between corresponding pixel intensities of an image. All the masks that are used for edge detection are also known as derivative masks. Because as we have stated many times before in this series of tutorials that image is also a signal so changes in a signal can only be calculated using differentiation.

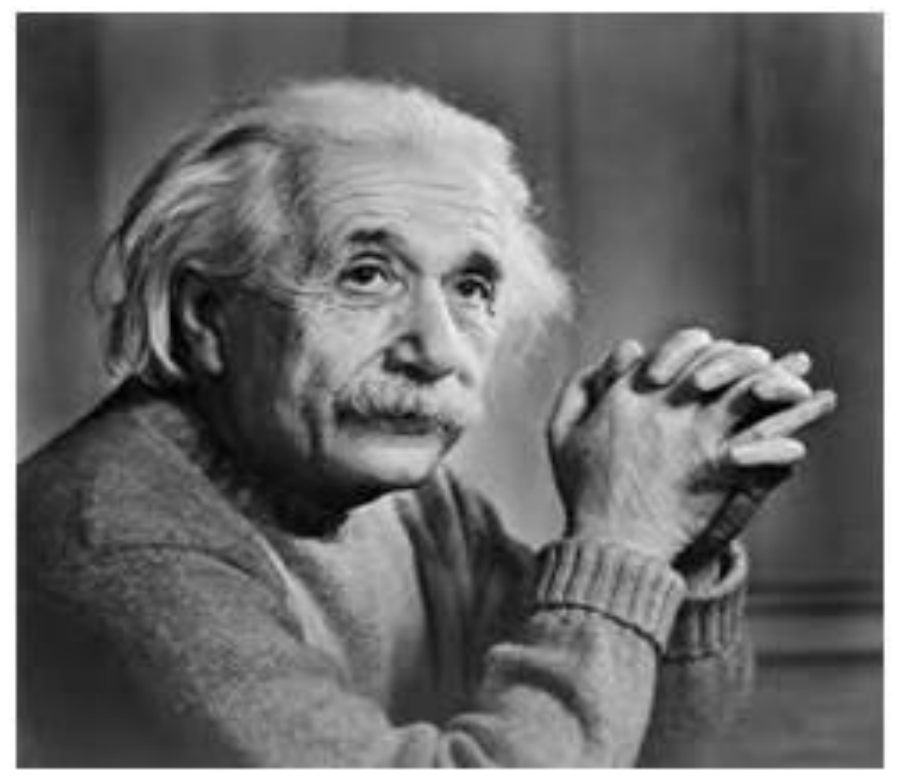

Fig. 2 Actual image 


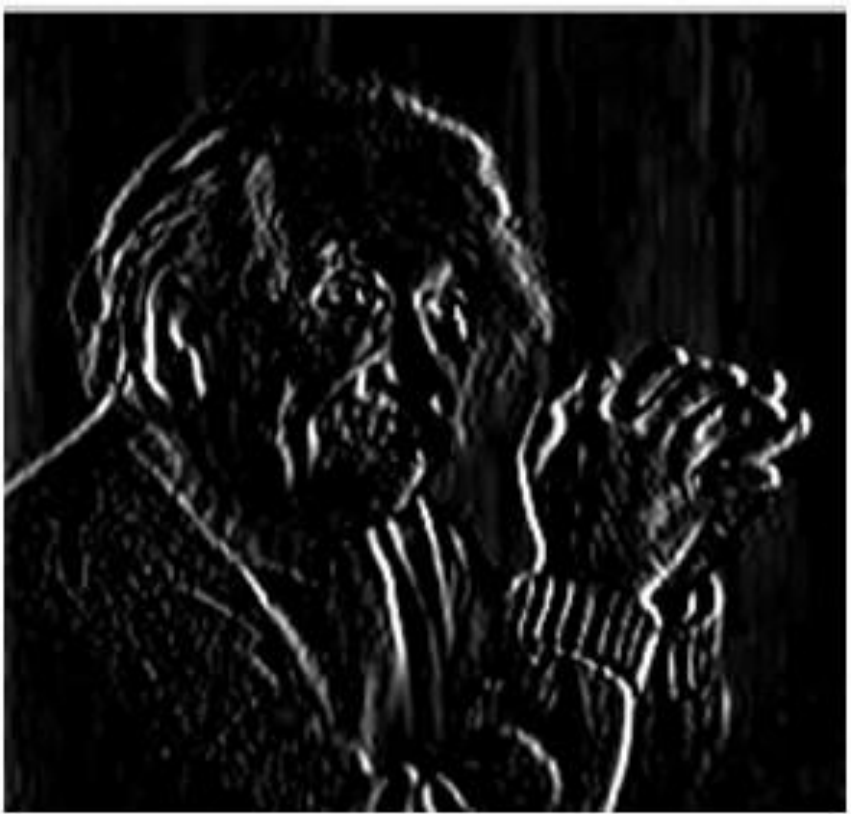

Fig. 3.a. Vertical mask

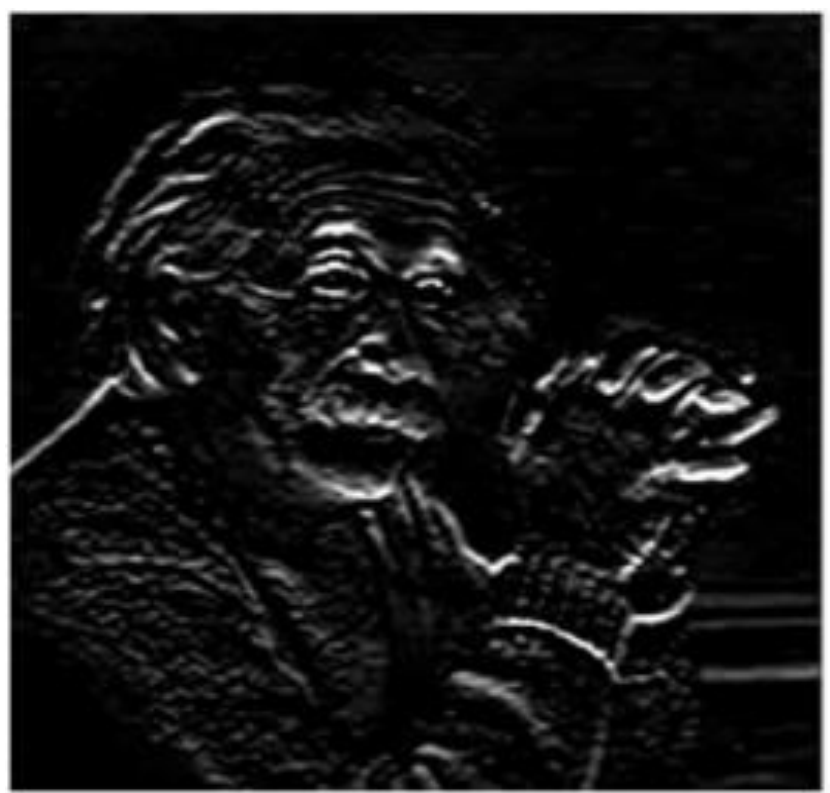

Fig. 3.b iii) Horizontal mask

\subsection{Extraction of Miniature Points and Matching}

After the extraction of edges, the points are marked in it. Those points which are detected after edge detection are known as miniature points. The miniature points that are extracted are compared with already stored images [6]. In order to find the matching process the correlation factor and the Euclidean distance has to be found out. Based on the tolerance value the matching results can be found out.

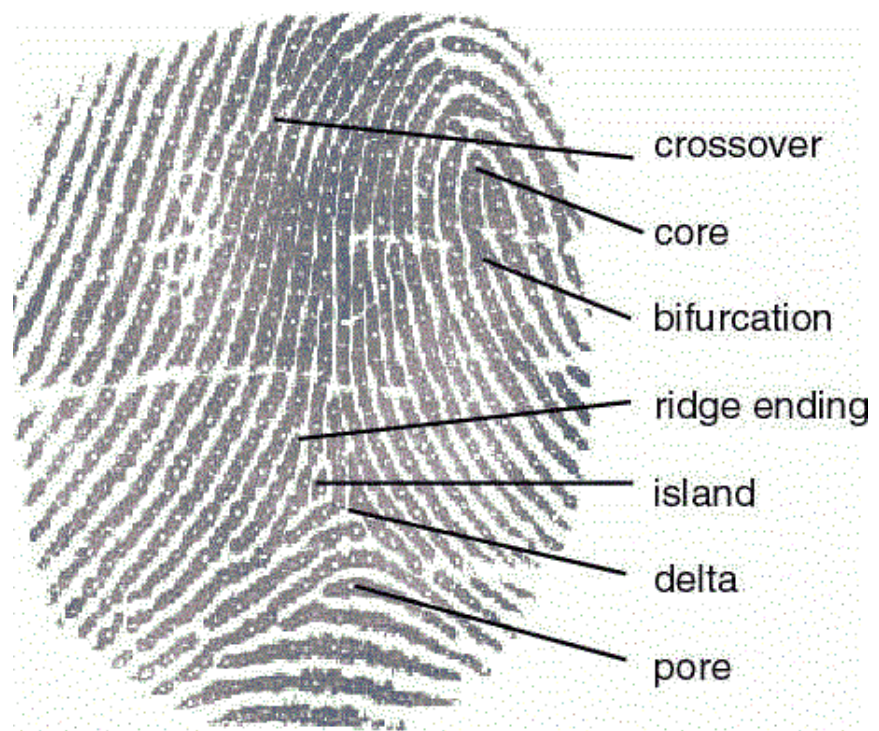

Fig. 4.a. minutiae before marking

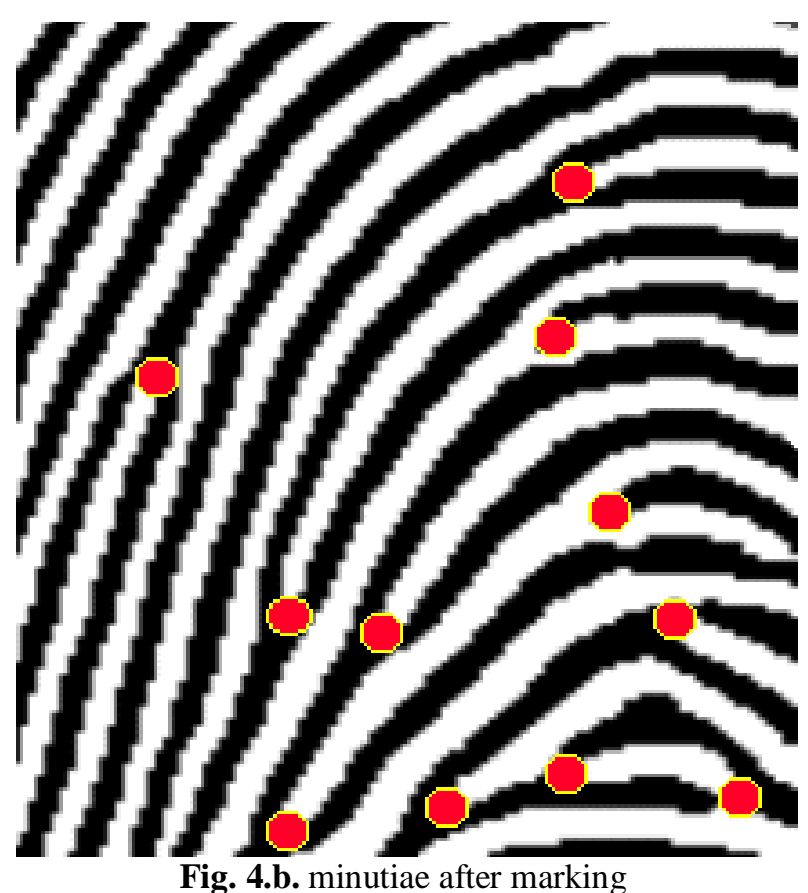

\section{GSM MODULE}

Global System for Mobile Communications (GSM), is a standard developed by the (European Telecommunications Standards Institute) ETSI in order to represent the protocols for $2 \mathrm{G}$ cellular networks used by mobile phones. We have majorly two types of SIM modules. They are SIM 300 and SIM 900. Here the SIM 300 is enough for our purpose. SIM 900 is used for some advanced purposes and it has many additional features. 


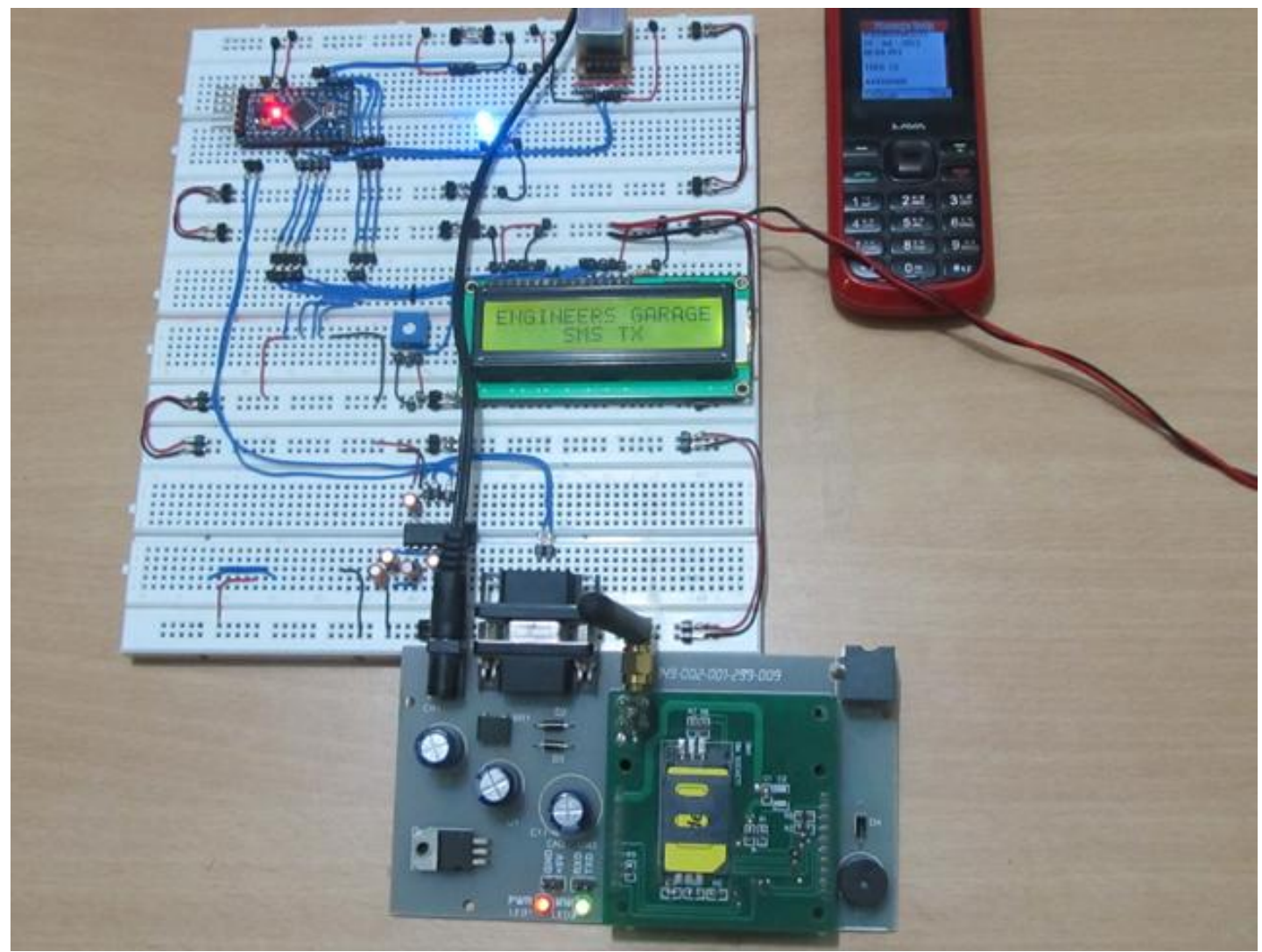

Fig. 5 Interface of GSM Module

The main purpose of the GSM in projects is to send and receive the messages. But not only that we can make a call and can we also browse using the GSM [7]. The GSM has the operating voltage of $12 \mathrm{v}$. It has mainly of three pins namely transmitter, ground and the receiver pin.

To perform these tasks, a GSM modem must support an "extended AT command set" for sending/receiving SMS messages. GSM is one of the most useful inventions in the modern world. It has many advantages than other technology standards. The Advantages of GSM are,

- Worldwide roaming

- Security

- Reasonable devices and facilities

- Extensive spectrums obtainable

\section{COMMUNICATION OF GSM WITH MICROCONTROLLER}

The result from the fingerprint module is taken and it is analyzed in the microcontroller. We use "ATMEGA 16" microcontroller in the paper. The result from the GSM module is received by microcontroller. If the already stored image in the memory and input image are matched then microcontroller will sent the control to the GSM module. The GSM will send the messages to respective parent's mobile numbers [8]. If the fingerprints are mismatched then the control signal will not be sent to the GSM module.
After some time interval the details of the students who were not present were taken. Those persons details were taken and message of "NOT PRESENT "is sent to their respective parent's mobile numbers. So, the parents may know about the student's presence immediately. The Students cannot forget this system easily.

\section{CONCLUSION}

The fingerprint-based attendance management system was implemented with Microsoft's C\# on the .NET framework and Microsoft's Structured Query Language (SQL) Server 2005 as the backend. The future work may consist of creating the database of students which contains the academic details of the students. We can send the academic details of the students periodically to theparent's mobile along with the attendance report. So in near future we can use finger vein recognition in this attendance system which very unique compared to the fingerprint authentication. The authentication can also be made as more secure by using human odor for the Security purpose.

\section{REFERENCES}

[1]. Murizah Kassim, Hasbullah Mazlan, Norliza Zaini, Muhammad Khidhir Salleh "Web-based Student Attendance System using RFID Technology" 2012 IEEE.

[2]. B. Rasagna, Prof. C. Rajendra "SSCM: A Smart Systemfor College Maintenance" International Journal of Advanced Research in Computer Engineering \& Technology, May 2012. 
[3]. LI Jian-po, ZHU Xu-ning, LI Xue, ZHANG Zhi-ming "Wireless Fingerprint Attendance System Based on ZigBee Technology" 2010 IEEE.

[4]. O. Shoewu, O.A. Idowu "Development of Attendance Management System using Biometrics" The Pacific Journal of Science and Technology, May 2012.

[5]. "How does a fingerprint sensor work" Online Available: http://wiki.answers.com/Q/How_does_a_fingerprintsensorw ork Accessed: Sept. 15, 2013].

[6]. "What is GSM?" Online Available: http://www.tutorialspoint.com/gsm/gsm_overview.htm[Acc essed: Sept. 17, 2013].

[7]. L. Rajasekar, S. Vivek "Wireless Fingerprint Attendance System using ZigBee Technology" International Journal of Power Control Signal and Computation (IJPCSC), Vol3. No1. Jan-Mar 2012.

[8]. Zatin Singhal, Rajneesh Kumar Gujral "Anytime Anywhere- Remote Monitoring of Attendance System based on RFID using GSM Network" International Journal of Computer Applications, February 2012. 